\title{
ANÁLISIS DEL PROCESO DE TRANSPORTE VIA SFT, DE LAS ERUPCIONES DEL 2014-2015 DEL VOLCÁN TURRIALBA
}

\author{
ANALYSIS OF TURRIALBA VOLCANO (ERUPTION 2014-2015) \\ TEPHRA TRANSPORT USING SFT
}

\author{
José Brenes-André \\ Red Ciudadana de Estaciones Meteorológicas, Apdo. 290-3015, Costa Rica \\ jbrenes54@gmail.com
}

(Recibido: 10/7/2017; aceptado: 8/12/2017)

\begin{abstract}
Resumen: Las tefras de las erupciones del volcán Turrialba (octubre 2014 a abril 2015) fueron analizadas por medio del SFT, pudiéndose determinar 5 procesos de transporte, que fueron analizados según los parámetros de Folk; utilizando la dispersión promedio en lugar de la desviación estandard, y . utilizando la dispersión por transporte g, según se describe en Wohletz et al. (1989). Los resultados obtenidos, no concuerdan con observaciones de campo. Como una alternativa, se examinó la dispersión por transporte $\mathrm{g}$ en función de la dispersión por fragmentación $\gamma$, encontrándose empíricamente que es posible definir la relación lineal $\Delta=\gamma-\mathrm{g}=\mathrm{m} \gamma+\mathrm{b}$ para cada uno de los 11 procesos de transporte identificados por medio de SFT para los dos Grupos de muestras. Los patrones evidentes entre varios de esos $\Delta$ sugieren que el valor de la pendiente $\mathrm{m}$ es una medida indirecta de la interacciones entre las partículas involucradas en el proceso de transporte. Esta observación sugiere que los números adimensionales (como el de Savage y el de Reynolds) y sus respectivos valores críticos asociados a las corrientes de densidad piroclástica explicarían la aparición de los diversos tipos de transporte detectados por SFT.

Palabras clave: SFT, volcán Turrialba, procesos de transporte de ceniza, dispersión por transporte de Wohletz, cambio de fase.
\end{abstract}

\begin{abstract}
SFT (Sequential fragmentation/transport) method was applied to recent fine tephra of october 2014 to april 2015 eruptions of Turrialba Volcano, to identify transport processes on Wohletz et al. (1989) transport dispersion (g). Results obtained that way do not match field observations. Hence, a new analysis is proposed based on an empirical linear relation between the fragmentation $(\gamma)$ and transport dispersion $(\mathrm{g})$, was devised. Provitional physical mechanisms are presented to help understand such an empirical lineal relationship.

Keywords: SFT, Turrialba volcano, ash transport processes, Wohletz transport dispersion, phase change.
\end{abstract}




\section{INTRODUCCIÓN}

Contrariamente a los eventos sísmicos que son más corrientes, las erupciones volcánicas son eventos aislados cuyo período de recurrencia fácilmente es de decenas y hasta centenas de años, lo que motiva a los investigadores a desarrollar métodos de análisis que extraigan la mayor información posible de los pocos eventos disponibles.

Según Sheridan (1971),las granulometrías polimodales son producto del traslape de complejos procesos que selectivamente concentran o remueven tefras de tamaños específicos durante el proceso de erupción y de transporte. En Visher (1969) y Eschner y Kircher (1984) se propone que en sedimentos subacuáticos las sub-poblaciones pueden ser asociadas a procesos de transporte y depositación por tracción, saltación, y suspensión.

Métodos como la sísmica, el sistema de posicionamiento global (GPS) y la gravimetría permiten la obtención de datos reiterada en el tiempo. Las limitantes financieras y de recurso humano se van reduciendo tanto por el abaratamiento de hardware, como por la disponibilidad de software capaz de manejar un creciente número de datos.

Otro método, el SFT (por su nombre en inglés: Sequential Fragmentation/Transport), que en principio puede aplicarse a un número arbitrario de granulometrías, tiene como inconveniente el estar basado en el concepto de fractal, cuyo uso no es muy común en el área geológica.

Como su nombre lo indica, el SFT analiza tanto el proceso de fragmentación, como el proceso de transporte de los productos. El análisis de fragmentación, basado en principios físicos inicialmente propuesto por Wohletz (1989) con una visión global, ha sido re-conceptualizado para obtener mayor información de cada granulometría. En Brenes (2013) esas metodologías globales son aplicadas a cada sub-población por separado; en Brenes y Alvarado (2013) se analiza el caso de las dispersiones positivas, asociándolas a procesos de fragmentación secundaria, en Brenes (2014) se demostró que la dispersión $\gamma=-0,5$ es el límite entre erupciones con procesos persistentes $(\gamma$ entre $-0,5$ y 0$)$ y aquellas con procesos antipersistentes
( $\gamma$ entre -1 y $-0,5)$, como se ha observado experimentalmente. Los llamados volcanes log-logísticos (tales como Colima, Soufriere y Erebus) también han sido analizados con ayuda del SFT (Brenes, 2016)

El análisis del transporte, por el contrario, se analiza con ayuda de una segunda dispersión (denominada g) propuesta en Wohletz et al. (1989) que es aproximada. Además, en la literatura a un mismo proceso de transporte se le ha asociado diferentes ámbitos de g. En el presente trabajo se analizarán algunas de las consecuencias de esta situación, proponiéndose una posible salida con base experimental.

\section{TIPOS DE TRANSPORTE Y LA SFT}

El estudio de los procesos de transporte usando SFT además de ser muy escasa es en parte contradictoria, como se verá más adelante. Por este motivo los dos grupos de muestras del volcán Turrialba serán analizados desde dos ópticas tradicionales: parámetros de Folk, y por dispersión por transporte g según Wohletz,así como por dos ópticas alternativas: dispersión promedio por transporte, por analogía a la propuesta de Folk; y una nueva óptica basada en la diferencia $\Delta=\gamma-\mathrm{g}$.

En un mismo evento eruptivo, es común encontrar que varias de las distribuciones de Weibull detectadas con ayuda del SFT tienen modas similares, producto quizá de la constancia de las propiedades físico-químicas del magma (Sheridan et al.,1987), y que pueden ser considerada como una sub-población.

En Wohletz et al. (1989) se propuso que los procesos de transporte cambian la forma de la distribución de Weibull de cada sub-población, lo que obliga a caracterizarlas con una nueva dispersión denominada " $\mathrm{g}$ ". La asociación de cada uno de los valores de $\mathrm{g}$ a uno, o varios, posibles procesos de transporte se efectuaría con ayuda de los criterios presentados en Wohletz et al. (1989) (Cuadro 1). En Brenes (2013) las granulometrías del Irazú (erupción 1963-65), con una resolución de $0,5 \Phi$, fueron analizadas calculando, en primer lugar, el valor de g para cada subpoblación. Luego, como una primera aproximación, 


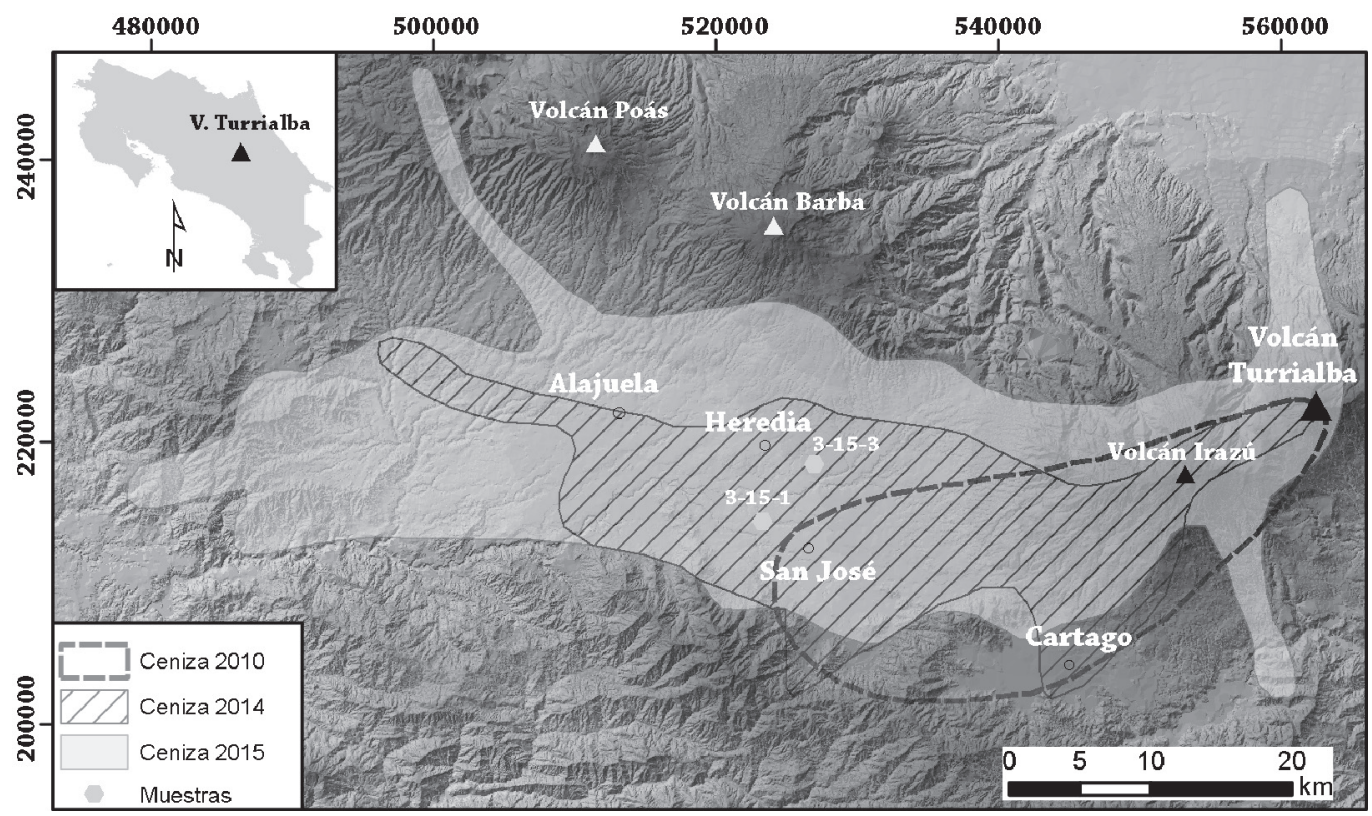

Fig. 1: Mapa de ubicación del volcán Turrialba, la distribución de las cenizas y de los aeropuertos afectados en el 2015: Aeropuerto Internacional Juan Santamaría (AIJS) y aeropuerto Tobías Bolaños (ATB). Se muestra también algunas muestras de ceniza recolectadas en el Valle Central.

los $\%$ en peso de las subpoblaciones asociadas a un mismo proceso fueron sumados, y finalmente $\operatorname{los} \%$ totales fueron revisados para determinar si reflejaban en términos generales el proceso de transporte en campo, según criterio experto. En este trabajo $\Phi=\log 2 \mathrm{~d}$, siendo d el tamaño del fragmento, expresado en milímetros.

Otra posible asociación entre g y procesos de transporte se puede lograr recurriendo a Sheridan et al. (1987), y discriminar así entre balística, saltación, tracción y suspensión. En algunos casos se podría proponer, siguiendo a Cas (1989), que cerca del cráter los depósitos pueden, por ejemplo, ser simultáneamente de caída y oleada, que él denominó como "depósitos de oleada modificados por caida"

En Wohletz (1998) se hace una propuesta muy diferente. Cada proceso de transporte es asociado a un único ámbito de tamaño. Así, se entendería por balística el transporte de las partículas grandes tales como lapilli, bloques y bombas (con tamaño de $\Phi<-4$, o sea mayores a $1,6 \mathrm{~cm}$ ) que son eyectadas con la velocidad suficiente para que su trayectoria no se afectada
Cuadro 1

Valores de $\mathrm{g}$ asociados a procesos provisionales de transporte, según Wohletz et al. (1989).

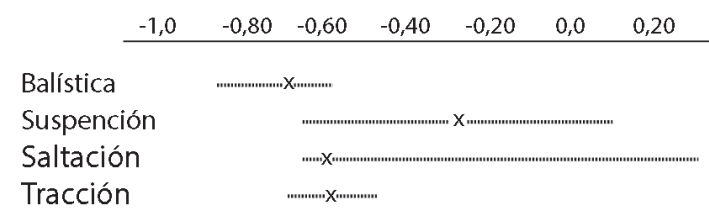

ni por la resistencia debida al aire ni por oleadas. La tracción sería solo posible en partículas de tamaño entre $-4 \Phi$ y $1 \Phi(0,5 \mathrm{~mm})$ que tendrían la suficiente velocidad terminal para depositarse en regiones concentradas, generalmente en la frontera de la capa basal, donde los esfuerzos cortantes son altos y las colisiones entre partículas son muy frecuentes. El transporte por saltación se produciría en partículas pequeñas $(1 \Phi$ a 4 $\Phi$, equivalentes a $0,064 \mathrm{~mm}$ ) con suficiente velocidad y espacio entre las partículas para que reboten, siguiendo una trayectoria parabólica, interaccionen con el sustrato hasta volver a rebotar. La suspensión solo afectaría a partículas 
muy pequeñas (diámetros menores a $4 \Phi$, o sea $0,064 \mathrm{~mm}$ ) que tendrían una velocidad terminal tan pequeña que los vórtices turbulentos presentes en las oleadas eviten que caigan, manteniéndose a flote.

Esta propuesta conlleva desestimar la nueva dispersión g como una caracterización de los procesos de transporte. Además, experimentalmente se encuentran granulometrías entre 1 y $5 \mathrm{~cm}$, en depósitos balísticos, de suspensión proximal (caída) y de tracciónsaltación (depósitos de densidad piroclástica) tal y como se ha observado y descrito a nivel mundial, y en caso particulares como el Arenal (Soto y Alvarado, 2006; Alvarado et al., 2006) (Guillermo Alvarado, comunicación personal).

\section{METODOLOGÍA}

Las muestras recolectadas de las erupciones del volcán Turrialba del 2014-2015 se clasificaron en dos grupos. El Grupo 1 (G1) está compuesto por muestras que se recolectaron pocas horas después del evento eruptivo de Octubre 2014 (muestras Tur-14-1A a Tur-14-6w) Información sobre las muestras de los eventos del 2014 .puede consultarse en el Appéndice 1. El grupo 2 (G2) lo componen muestras de eventos ocurridos en el 2015. La localización de las muestras que componen el grupo 2 (G2) se muestran en la figura 2.

Tefras del volcán Irazú (Brenes, 2013) como del Turrialba (Alvarado et al., 2016) fueron analizadas con ayuda del método SFT (Los Alamos National Laboratory computer code LA-CC 99-29, versión 2.22.0170, http:// en.freedownloadmanager.org/Windows-PC/ KWare-SFT-FREE.html, 2015, Los Alamos, New Mexico). Como se ha observado en otros casos, se encontró que en muestras de un mismo ciclo eruptivo se repiten ámbitos de tamaño, lo que refleja procesos físicos subyacentes en esos procesos (Sheridan et al.,1987; Wohletz et al., 1989).

La resolución de $1 \Phi$ de las granulometrías amplía el porcentaje del residuo: entre 3\% y $13 \%$ para las muestras tomadas pocas horas después de la erupción (Tur14-1a a Tur-14-6w), y entre 5\% y $10 \%$ para las muestras tomadas varios meses después del evento, tal el caso de Tur-12-3-15-1; Tur-12-3-15-3 (ambas del 12 Marzo 2015), Tur19-3-15; 1030-AM1 y 12-09A1.

\section{Análisis de muestras Grupo 1}

El transporte por suspensión es fácilmente asociable a aquellas muestras recolectadas a una decena de kilómetros del cráter. En el otro extremo, los fragmentos recolectados cerca de la fuente generalmente serían asociados a procesos balísticos. Sin embargo, las muestras del Grupo 1 cercanas a la fuente tienen mayoritariamente un tamaño que excluyen el transporte balístico, por lo que en este trabajo se asociarán a una mezcla en proporciones variables de tracción y de saltación (oleada), pues considerarse que los modelos nopermiten discriminar entre ambos procesos.

Para ser completos, en este trabajo, las granulometrías se analizarán según: a) los parámetros de Folk; b) la dispersión g promedio, obtenida a partir de SFT , c) las dispersiones g asociadas a cada una de las distribuciones de Weibull en que se descomponen con ayuda de SFT, y una nueva óptica basada en la diferencia $\Delta=\gamma-\mathrm{g}$., como se indicara líneas atrás.

$\mathrm{Su}$ análisis se llevará a cabo utilizando solo 3 procesos de transporte: el de suspensión (distinguible por la gran distancia a que fueron recolectadas las muestras), el de balísitica (por la cercanía y tamaño de los fragmentos), como end members (las colas tiene información, Doeglas, 1946) y uno intermedio de oleada, entendido como una combinación en proporciones variables de tracción y saltación. Se carece de información suficiente sobre si el transporte se dio en un medio líquido o gaseoso, y así poder valorar las variaciones locales de la velocidad en el aire, que son más importantes que las del agua al mover un grano. Igualmente no es posible evaluar si prevalecieron movimientos back and forth, o por el contrario se dio un movimiento neto unidireccional. A pesar de ello, se concuerda con 


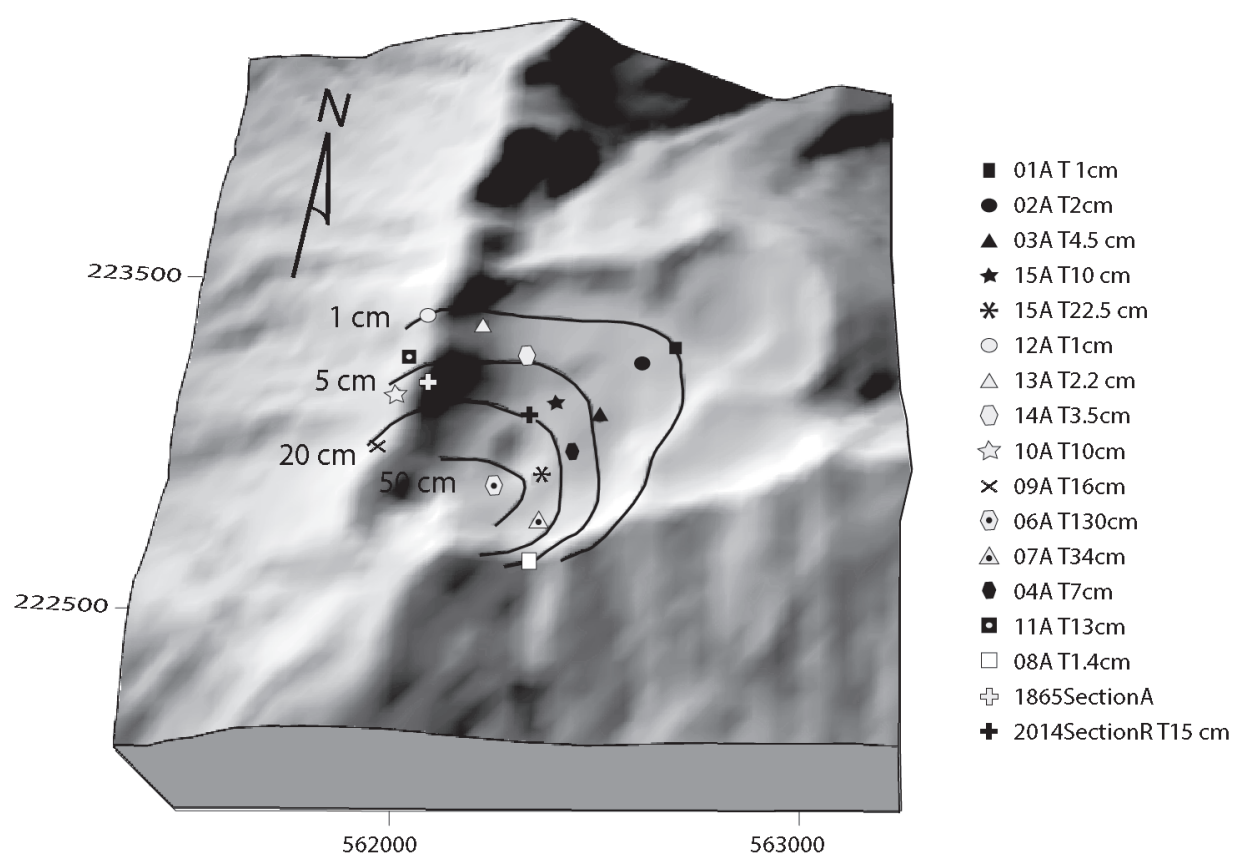

Fig. 2: Localización de las muestras del Grupo 2. Las curvas de trazos corresponden al espesor de isopacas calculadas.

Arthur et al. (1986) en que se requiere bastante tiempo para que las masas transportadas por un agente pierdan completamente las características asociadas a él luego de empezar a ser transportada por otro agente.

\section{Parámetros de Folk}

Los parámetros de Folk fueron obtenidos con ayuda del programa SFT. Aunque los parámetros estadísticos calculados según Folk son comunes en la literatura, estos deben, sin embargo, ser tomados con cautela. Si bien no se utilizaron intervalos menores a $1 \Phi$, se advierte que no se evaluó si el ámbito de los parámetros estadístico es lo suficientemente grande, como previenen Swan et al. (2006).

En Cuadro 2 se muestran los valores de desviación estandard, asimetria y curtosis versus la mediana, tanto para oleada como para supensión.

En la figura 3 se observa que las desviaciones standard permiten diferenciar entre oleadas (con valores entre 1 y 2,5 , y medianas en el ámbito de
0 a 2,5). y suspensiones (mediana mayor a 2,5, con desviaciones alrededor de 0,5 ). Ni la asimetría ni la curtosis parecen discriminar tan claramente entre esos dos tipos de transporte, aunque vale la pena mencionar que las asimetrías negativas son muy comunes en depósitos de oleadas y flujos piroclásticos.

\section{Análisis por dispersión g promedio}

El programa SFT permite calcular una dispersión de transporte g promedio para toda la granulometría, lo que sugiere re-hacer el análisis anterior utilizando la moda y la dispersión promedio g en lugar de la mediana y la desviación estándard de Folk.

Por lo alejado del cráter, las muestras recolectadas en Pavas $(g=-0,7)$, Santo Domingo $(g=$ $-0,48)$ y la cerca del Mirador $(\mathrm{g}=-0,57)$ necesariamente estarían asociadas a procesos de suspensión, por lo que valores de g iguales o mayores a $-0,7$ o mayor se asociarían a suspensión. Los restantes se asocian a oleadas, pues la distancia del cráter a que fueron recolectadas hace poco 
Cuadro 2

Análisis del grupo 1, según SFT. El grado de selección se asignó según Cas y Wright (1987). Ca-ole: caída modificada por oleada piroclástica; Ole-ca: oleada modificada por caída. MB: muy buena, B: buena y P: pobre. Plati: platicúrtica, Meso: mesocúrtica, Lepto: leptocúrtica.

\begin{tabular}{|c|c|c|c|c|c|c|c|c|}
\hline Muestra & $\begin{array}{l}\text { Depósito } \\
\text { asociado }\end{array}$ & Mediana & $\begin{array}{c}\text { Desviación } \\
\text { estándar } \\
\text { Folk }\end{array}$ & $\begin{array}{l}\text { Grado de } \\
\text { selección }\end{array}$ & Asimetría & Curtosis & Clase & $\begin{array}{l}\text { Residuo } \\
\text { según SFT }\end{array}$ \\
\hline Tur14-1a & Suspen. & 1,25 & 1,87 & B & $-0,17$ & 0,69 & Plati & $8,8 \%$ \\
\hline Tur14-1b & Suspen & 2,55 & 0,92 & MB & $-0,05$ & 0,81 & Plati & $9,35 \%$ \\
\hline Tur14-2 & Oleada & 1,45 & 1,25 & B & 0,06 & 0,98 & Meso & $6,66 \%$ \\
\hline Tur14-3 & Oleada & 1,15 & 1,43 & B & $-0,28$ & 1,25 & Lepto & $4,49 \%$ \\
\hline Tur14-4 & Oleada & 2,15 & 1,23 & B & $-0,08$ & 0,84 & Plati & $9,69 \%$ \\
\hline Tur14-5 & Ole-Sus & 2,0 & 1,09 & B & 0,07 & 0,88 & Plati & $6,46 \%$ \\
\hline Tur14-6w & Ole-Sus & 0,05 & 1,83 & B & 0,03 & 0,98 & Meso & $3,26 \%$ \\
\hline Tur14-7 & Sus-Ole & 2,45 & 1,09 & B & $-0,11$ & 0,90 & Plati & $12,82 \%$ \\
\hline Tur19-3-15 & Suspen & 3,37 & 0,51 & MB & $-0,25$ & 1,1 & Meso & $21,40 \%$ \\
\hline $\begin{array}{c}\text { Tur12-3- } \\
15-1\end{array}$ & Suspen & 2,86 & 0,59 & MB & 0,2 & 0,81 & Plati & $8,85 \%$ \\
\hline $\begin{array}{c}\text { Tur12-3- } \\
15-3\end{array}$ & Suspen & 2,54 & 0,55 & MB & 0,25 & 1,06 & Meso & $4,31 \%$ \\
\hline $\begin{array}{l}\text { 10-30- } \\
\text { AM1 }\end{array}$ & Sus-Ole & 1,16 & 1,6 & B & $-0,08$ & 0,96 & Meso & $5,28 \%$ \\
\hline 12-09A1 & Sus-Ole & 1,26 & 2,23 & $\mathrm{P}$ & $-0,28$ & 0,69 & Plati & $10,35 \%$ \\
\hline
\end{tabular}

probable que influyera el transporte balísitico. Los resultados se muestran en el Cuadro 3, en el que se incluye el porcentaje asociado a cada tipo de transporte, denominados \%Ole y \%Suspen.

Esta aproximación al problema, sin embargo, presenta una serie de inconsistencias. Por ejemplo, la muestra Tur14-1b debería ser asociada a suspensión (dispersión promedio de $\mathrm{g}=-0,68$ ) (como se consideró en Folk), pero presenta un $72 \%$ de componente de oleada.

Además, entre mayor sea la dispersión por transporte g más seleccionada sería la muestra (Wohletz et al. ,1989), algo que no se cumple con la muestra recolectada en Pavas $(g=-0,7)$, a varios kilómetros del cráter. Esta debería ser más seleccionada que la recolectada al borde del cráter cerca del Mirador, $(\mathrm{g}=-0,57)$, aunque la diferencia podría deberse a contaminación.

La dispersión promedio arroja también otros resultados contradictorios al aplicarsela a dos muestras de ceniza recolectadas en sitios muy cercanos; una en la superficie acanalada de un

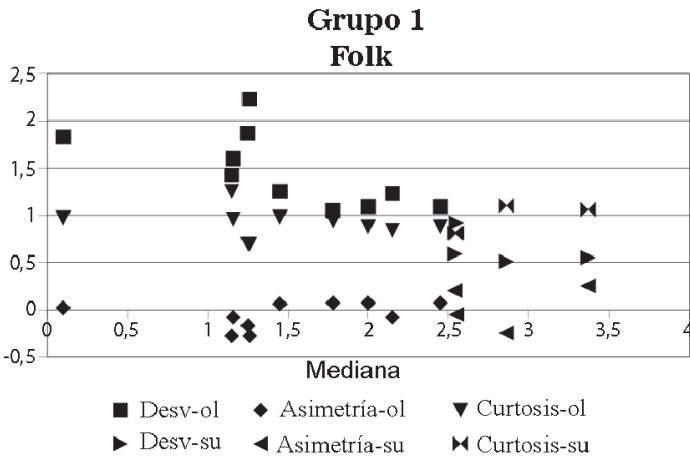

Fig. 3: Valores (según Folk) de la desviación estandard, la asimetría y la curtosis para las dos clases: oleada (ol) y suspensión (su), versus la mediana, usando los valores del Cuadro 2.

tronco caído, a medio metro de altura (Tur141a con una masa: $207,25 \mathrm{~g}$ ), y la otra sobre las hojas de un plantío de papas (Tur14-1b: 37,6 g). Ambas son platicúrticas, con oblicuidad o asimetría (skewness) negativa, es decir, presentan un reducido grado de concentración alrededor de los valores centrales de la variable. Con mucha 
Cuadro 3

Valores de la dispersión g promedio para las muestras del Grupo 1.

\begin{tabular}{cccccc}
\hline Muestra & Disp. Prom. & Transporte & Localidad & \% Oleada & \% Caida \\
\hline Tur12-3-15-2 & $-0,9$ & Oleada & Paredes & 100 & \\
12-09A1 & $-0,83$ & Oleada & & 100 & \\
Tur14-7 & $-0,75$ & Oleada & & 100 & \\
Tur14-6 & $-0,84$ & Oleada & & 95 & 5 \\
Tur14-2 & $-0,86$ & Oleada & & 93 & 7 \\
Tur14-1a & $-0,73$ & Oleada & & 82 & 18 \\
Tur14-4 & $-0,81$ & Oleada & & 79 & 21 \\
10-30AM1 & $-0,81$ & Oleada & & 76 & 24 \\
Tur14-1b & $-0,68$ & Caida & & 72 & 28 \\
Tur14-5 & $-0,78$ & Oleada & & 67 & 33 \\
Tur14-3 & $-0,77$ & Oleada & & 51 & 49 \\
Tur12-3-15-1 & $-0,7$ & Caida & Pavas & & 100 \\
Tur19-3-15 & $-0,57$ & Caida & Mirador & & 100 \\
Tur12-3-15-3 & $-0,61$ & Caida & Sto. Domingo & & \\
\hline
\end{tabular}

probabilidad, la muestra recolectada en la oquedad (Tur14-1a), representa la suma de eventos acaecidos entre el 29 de octubre hasta el amanecer del 1 de noviembre del 2014, mientras que la muestra sobre las hojas de papa (Tu14-1b), representan únicamente el último evento, dado que las lluvias debieron de haber lavado las cenizas previas. Al poseer la Tur14-1a una selección regular a buena, se esperaría que su correspondiente $g$ fuera mayor (Wohletz et al., 1989) que el correspondiente de la Tur14-1b, opuesto a lo que se obtuvo: un valor de $\mathrm{g}=-0,73$ para la muestra Tur14-1a, menor al valor de $\mathrm{g}=-0,68$ para la muestra Tur14-1b.

\section{Análisis por dispersión polimodal}

La sustitución de la desviación estandard por la dispersión promedio g es una posición híbrida que en lo fundamental mantiene la posición de Folk, en lugar de adoptar la visión de SFT. Según Sheridan et al. (1987) a cada una de las sub-poblaciones identificadas al aplicar SFT a una granulometría específica debe asociarse un proceso de transporte específico. Por lo tanto, cada sub-población debería entonces ser analizada utilizando su valor de dispersión de transporte g asociado. Empíricamente se encuentra que las sub-poblaciones de todas las granulometrías de las muestras de los grupo 1 y 2 tienden a agregarse alrededor de valores de moda similares. Por ello, en lo que sigue en este trabajo se denominará proceso de transporte a cada uno de esos agregados de subpoblaciones.

Siguiendo esa lógica, tres procesos pueden identificarse (Fig. 4): el proceso 5 con sub-poblaciones con modas entre 3 a $4 \Phi$ y valores de g entre $-0,8$ y $-0,3$, que podría asociarse a transporte por suspensión. El proceso 1 compuesto por sub-poblaciones con modas en el ámbito de $-4 \Phi$ a $-2 \Phi$ y valores de $g$ entre $-0,9$ y $-0,8$, que se asociaría con transporte balístico. Y tres procesos con sub-poblaciones en el ámbito de $-2 \Phi$ a $2 \Phi$. Los procesos 2 y 4 , con valores de g entre $-0,9$ y $-0,8$, se asociarían a oleadas por su tamaño. El proceso 3, por tamaño debería también ser considerado como una oleada, pero los valores de g sugieren algunos casos de oleada modificada por suspensión, situación para la cual no se puede presentar ninguna explicación. 


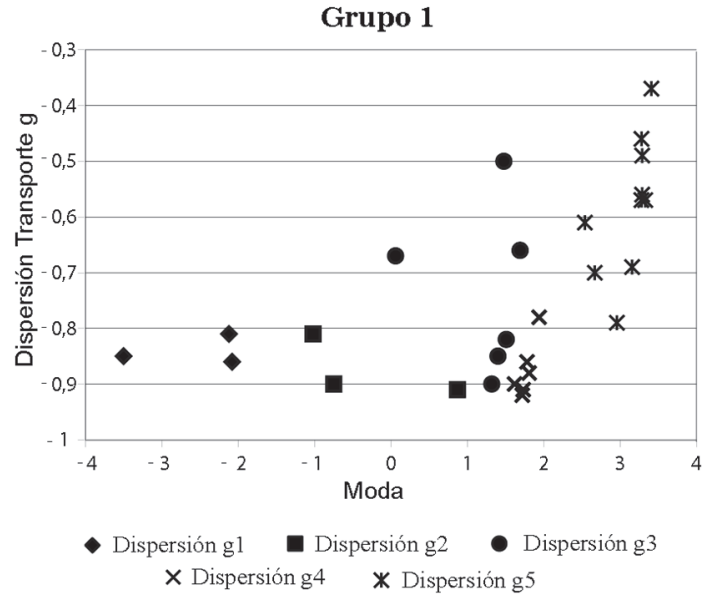

Fig 4: Gráfico del valor de dispersión de cada sub-población del Grupo 1 en función de la moda respectiva.

En el cuadro 4 se destacan los 3 casos con dispersión positiva, que no se asociaron a ningun tipode transporte.

\section{Análisis del Grupo 2}

Las muestras del Grupo 2 fueron todas recolectadas alrededor del cráter a inicios del 2015, por lo que pueden incluir tefras retrabajadas de las erupciones de finales de 2014. En la figura 5 se presentan los resultados obtenidos por SFT en la opción polimodal.

En este caso las sub-poblaciones con modas cercanas a $3 \Phi(0,125 \mathrm{~mm})$ y valores de $\mathrm{g}$ entre $-0,8$ y $-0,3$, conforman el proceso 5 que se asocia a suspensión. La mayoría de las distribuciones entre $-3 \Phi(8 \mathrm{~mm})$ y $2 \Phi(0,25 \mathrm{~mm})$ presentan valores de $\mathrm{g}$ entre -1 y $-0,8$ que pueden asociarse a procesos de transporte de oleada.

Las sub-poblaciones con dispersiones $\mathrm{g}$ entre $-0,9$ y $-0,6$ y modas entre $1,5 \Phi$ y $2,5 \Phi$ conforman el proceso 4 e incluirían algunos casos productos de oleadas afectadas por suspensión.

Todas, excepto una, de las sub-poblaciones con moda en el ámbito de $-5 \Phi$ a $-3 \Phi$ y dispersión g entre $-0,7$ y $-0,3$ se agrupan en el proceso 1 asociado a transporte balístico. La excepción corresponde al 24\% de la muestra 5A2 (moda -3,56 $\Phi$ y g -0,86), que se considera como balística
Cuadro 4

Sub-poblaciones asociadas a fragmentación secundaria.

\begin{tabular}{cccc}
\hline Muestra & Dispersión & Moda & \% en peso \\
\hline 10-30-AM1 & 0,01 & $-2,63$ & 2 \\
10-30-AM1 & 0,01 & 3,34 & 6 \\
Tur14-2 & 0,01 & 3,35 & 5 \\
\hline
\end{tabular}

modificada por oleada dada la proximidad con el siguiente grupo. Las observaciones anteriores se resumen en el cuadro 5.

En el Grupo 2 se encontraron 5 subpoblaciones con dispersión por fragmentación $\gamma$ positiva, muy cercanas a cero (Cuadro 6), que en Brenes y Alvarado (2013) fueron explicadas como fragmentaciones secundarias. Llama la atención que en para transporte el programa SFT genera también un $\mathrm{g}=0,01$, al que no puede asociarse a ningun tipo de transporte

\section{Resumen de los datos}

Los resultados de cada una de las opciones se resumen en el cuadro 7 para facilidad del lector.

Los datos concuerdan parcialmente con aquellos propuestos en Wohletz et al. (1989). Experimentalmente para balística el tamaño mínimo es la mitad, y el máximo es el doble del propuesto por Wohletz. Aunque el ámbito de g para balística, y prácticamente el encontrado para suspensión caen dentro del propuesto por Wohletz, el ámbito de g encontrado para las oleadas es totalmente diferente de los propuestos para tracción y saltación.

\section{ANÁLISIS DEL TRANSPORTE DESDE UNA NUEVA ÓPTICA}

En Sheridan et al. (1987) se proponen 3 posibles mecanismos que den origen a las subpoblaciones detectadas con ayuda del SFT: a) que fueran heredadas de la fragmentación inicial del magma y las rocas; b) que sean producto del tipo de clasto y su densidad, y c) que cada sub-población sea resultado de un proceso de transporte diferente. En Wohletz et al. (1989) 
Grupo 2

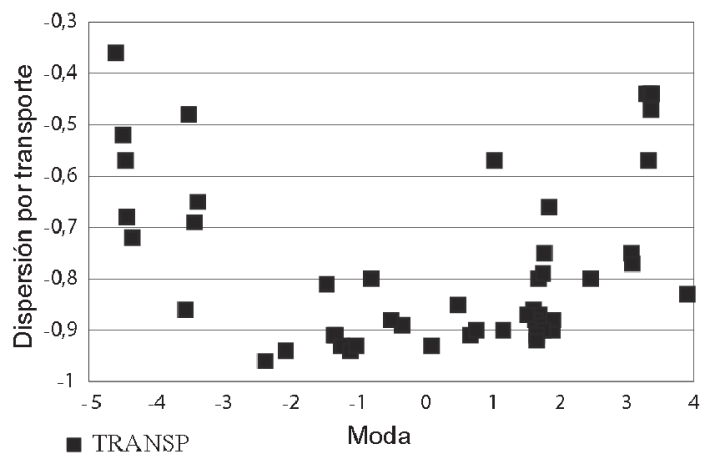

Fig 5: Gráfico del valor de dispersión de cada sub-población del Grupo 2 en función de la moda respectiva

se propone que, para cada sub-población, el proceso de transporte modifica la dispersión por fragmentación $\gamma$, dando lugar a la dispersión por transporte g. Así, por ejemplo, una sub-población con una dispersión por fragmentación de $\gamma=-0,91$ se transforma en una con dispersión $\mathrm{g}=-0,95$ al incluir el efecto del transporte. Los valores fueron obtenidos a partir del programa SFT que incluye dos opciones de análisis: solo fragmentación, y fragmentación/transporte. La definición de $\mathrm{g}$ sugiere que el proceso de transporte está entonces relacionado con la diferencia $\Delta=\mathrm{g}-\gamma$.

La idea subyacente a SFT es el proceso de conmunición que se da, por ejemplo, en un molino de bolas, que consiste en un cilindro cerrado conteniendo algunas esferas de metal muy rígido y fragmentos grandes del material a ser molido. Mientras el cilindro rota las bolas van golpeando el material, fragmentando. Ya que el tamaño de las esferas no cambia, los fragmentos de material más pequeños que cierto tamaño tendrán una mayor probabilidad de no ser afectados al poder alojarse en los espacios vacíos que se dan cuando varias de esas esferas se tocan, causando que la distribución por tamaño resultante de este proceso de molienda muestre una asimetría hacia los finos. Además, la cantidad de material contenido dentro del cilindro no cambiará durante todo el proceso, según el principio Newtoniano de conservación de masa. Como se muestra en Brown (1983), es posible formular ese principio como una ecuación integral que, unida al concepto de fragmentación
Cuadro 5

Muestras del grupo 2 distribuidas según el tipo de transporte.

\begin{tabular}{cccc}
\hline Muestra & $\%$ Balística & $\%$ Oleada & $\begin{array}{c}\% \\
\text { Suspensión }\end{array}$ \\
\hline 1A & & 83 & 15 \\
$2 \mathrm{~A} 1$ & 5 & 95 & \\
$2 \mathrm{~A} 2$ & & 31 & 69 \\
$3 \mathrm{~A} 2$ & 58 & 41 \\
$4 \mathrm{~A} 1$ & 44 & 53 & \\
$4 \mathrm{~A} 2$ & & 80 & 15 \\
$5 \mathrm{~A} 1$ & 76 & 22 & \\
$5 \mathrm{~A} 2$ & 24 & 64 & 12 \\
$5 \mathrm{~A} 3$ & 15 & 48 & 37 \\
$6 \mathrm{~A} 3$ & 5 & 95 & \\
$7 \mathrm{~A} 1$ & 32 & 68 & \\
$7 \mathrm{~A} 2$ & & 96 & 4 \\
$7 \mathrm{~A} 3$ & & 100 & \\
$10 \mathrm{~A} 1$ & 5 & 89 & 5 \\
$10 \mathrm{~A} 2$ & & 83 & 11 \\
$11 \mathrm{~A}$ & & 48 & 22 \\
$13 \mathrm{~A} 1$ & 46 & & \\
\hline
\end{tabular}

fractal, tiene como solución matemática una distribución de Weibull. De esta manera, la dispersión por fragmentación $\gamma$ tiene una base científica sólida.

Por el contrario, la dispersión g es propuesta, por analogía, como la dispersión de una nueva distribución Weibull inicialmente generada por conmunición dentro del cráter, y luego modificada por el proceso de transporte fuera de él. En opinión del autor, esta posterior fragmentación y/o elutriación se da en condiciones en que la masa no se conserva, por lo cual no existe garantía de que la distribución continue siendo una Weibull. Aún en casos más restrictivos en que la masa que entra a un volumen de control sea tal que supla la que se pierda por asentarse en el suelo, conservando la masa del flujo conforme se vaya moviendo, la objeción sigue aplicando pues la distribución por tamaño puede variar. Podría, sin embargo, plantearse que la distribución Weibull inicial no queda fuertemente afectada por el transporte si las muestras son recolectadas cerca de la fuente, o bien por 
Cuadro 6

Muestras del grupo 2 que presentan alguna sub-población con un $\mathrm{g}$ mayor a cero.

\begin{tabular}{cccc}
\hline Muestra & Dispersión & Moda & \% en peso \\
\hline 4A2 & 0,02 & 3,35 & 5 \\
4A1 & 0,01 & 3,34 & 3 \\
5A1 & 0,01 & 3,38 & 1 \\
13A1 & 0,01 & 3,36 & 4 \\
\hline
\end{tabular}

que la intensidad del mismo no la afecta apreciablemente a pesar de la distancia recorrida. Los flujos piroclásticos mantendrían en cierto grado memoria de la dispersión por fragmentación $\gamma, \mathrm{y}$ una expansión lineal $\Delta=\mathrm{m} \gamma+\mathrm{b}$ refejaría en buena medida esta situación.

Los dos conjuntos de muestras de erupciones del volcán Turrialba, recolectadas todas cerca de la fuente, algunas de ellas unas pocas horas después de ser eyectadas (Grupo 1), y otras conformando la suma de varios eventos que se sucedieron en un período prolongado (Grupo 2) posiblemente en condiciones cambiantes en el tiempo, permiten poner a prueba estas ideas.

Los datos iniciales utilizados para calcular $\Delta=\mathrm{g}-\gamma$ se obtuvieron siguiendo un estricto protocolo. Inicialmente, cada una de las granulometrías fue repetidamente analizada con el programa SFT, utilizando la opción de solo fragmentación, hasta reducir al máximo posible el $\%$ de residuo. Posteriormente, la descomposición de la granulometría original fue optimizada utilizando la opción fragmentación/transporte, usando como punto de partida los parámetros obtenidos en la primera etapa. Por último se examinó la variación de $\Delta=\mathrm{g}-\gamma$ respecto a $\gamma$, diferenciando cada uno de los posibles procesos involucrados, en analogía con lo propuesto en Brenes (2013).

En el gráfico de $\Delta=\mathrm{g}-\gamma$ respecto a $\gamma$ se observa que la recta de mejor ajuste para los procesos 2, 3 y 4 son muy parecidas entre sí con pendientes intermedias entre la del proceso 1 (la menor) y la del proceso 5 (la mayor). Se advierte que, por coherencia, en este gráfico no se incluyeron dos casos en que $\Delta=0$ (según la precisión del programa) por implicar que el transporte no afectó la fragmentación inicial, aunque si incluyeron 3 casos en que $\Delta$ está cerca de 0 . Otros 7 casos que tampoco fueron incluidos son aquellos con $\gamma=0,01$, asociados a fragmentaciones secundarias, cuyo respectivo valor de g también resultó 0,01 .

Comparándo la figura $6 \mathrm{~b}$ con la Figura 6a, utilizado para definir que sub-poblaciones conforman cada proceso, se nota que las correspondientes pendientes de los 5 procesos son similares cualitativamente. De hecho, en lo que sigue se mostrará que del gráfico de $\Delta=\mathrm{g}-\gamma$ respecto a $\gamma$ se puede obtener mayor información, pues el razonable ajuste $\Delta=\mathrm{m} \gamma+\mathrm{b}$ permite usar la pendiente $\mathrm{m}$ como una medida de las colisiones entre los fragmentos, y el intercepto b como una medida de la interacción de los fragmentos con el sustrato.

Los resultados se resumen en el cuadro 8 , en el que la asignación provisional de los procesos de transporte se efectuó usando de guía a Sheridan et al. (1987) y Wohletz et al. (1998). La pendiente $\mathrm{m}$ y el intercepto $\mathrm{b}$ corresponden a la $\operatorname{recta} \Delta=\mathrm{m} \gamma+\mathrm{b}$.

Cuadro 7

Clasificación de los procesos de transporte según el tamaño, Wohletz et al. (1998) y la dispersión por transporte g según Wohletz et al. (1989), para los Grupos 1 y 2.

\begin{tabular}{ccccc}
\hline Grupo & Balística & Oleada & Suspensión & Criterio \\
\hline 1 & $-4 \Phi$ a $-2 \Phi$ & $-2 \Phi$ a $2 \Phi(4 \mathrm{a} 0,25 \mathrm{~mm})$ & $3 \Phi$ a $4 \Phi(0,25 \mathrm{a} 0,064 \mathrm{~mm})$ & Diámetro \\
2 & $-6 \Phi$ a $-3 \Phi$ & $-3 \Phi$ a $3 \Phi(8 \mathrm{a} 0,125 \mathrm{~mm})$ & $3 \Phi$ a $4 \Phi(0,125 \mathrm{a} 0,064 \mathrm{~mm})$ & Diámetro \\
Wholetz & $<-4 \Phi$ & $-0,9$ a $-0,8$ & $>4 \Phi$ & Diámetro \\
1 & $-0,9$ a $-0,8$ & -1 a $-0,8$ & $-0,8$ a $-0,3$ & Dispersión $\mathrm{g}$ \\
2 & $-0,7$ a $-0,2$ & $-0,7$ a 0,3 Saltación & $-0,6$ a $-0,3$ & Dispersión $\mathrm{g}$ \\
Wholetz & $-0,9$ a $-0,6$ & $-0,7$ a 0,1 & Dispersión $\mathrm{g}$ \\
\hline
\end{tabular}



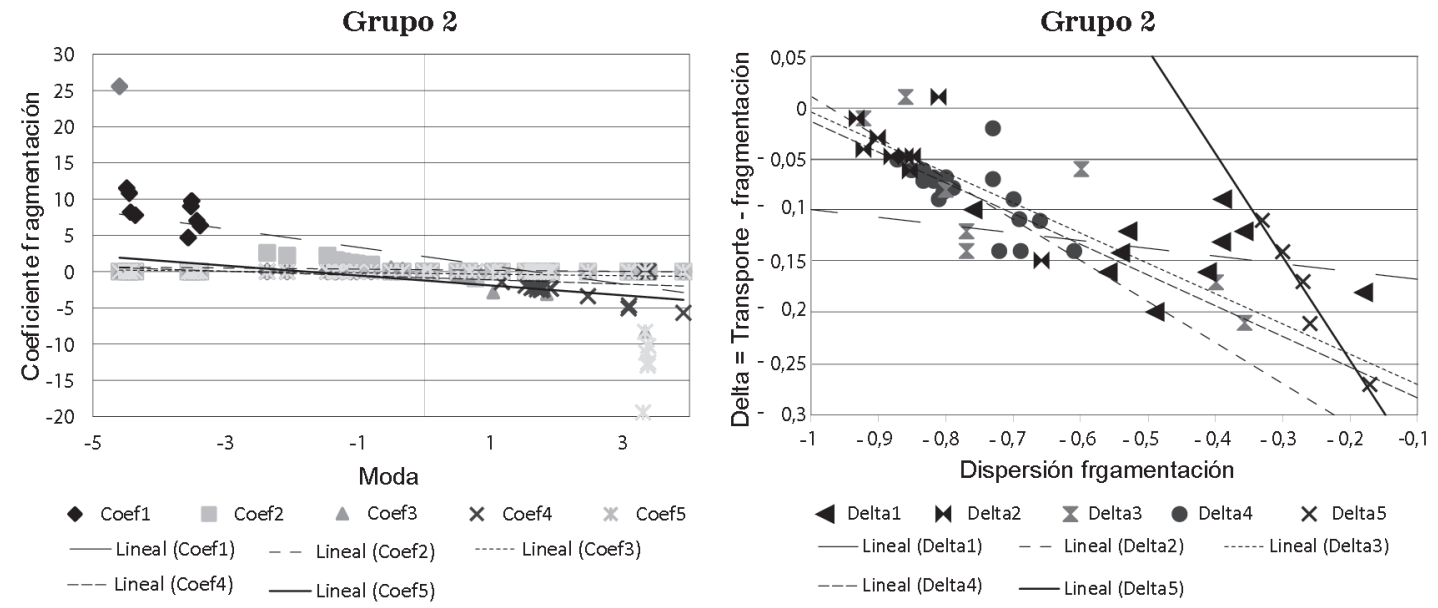

Fig. 6b: Gráfico de $\Delta$ respecto a $\gamma$ para todas las muestras del Grupo 2, que evidencia la posibilidad de definir 5 procesos de transporte via ajustes lineales.

\section{DISCUSIÓN}

Los criterios para la asignación del tipo de transporte propuestos en Wohletz et al. (1998) y en Sheridan et al. (1987) no siempre coinciden. Según Wohletz para el Grupo 1 los procesos 1 y 2 estarían asociados a tracción: y los 3 y 4 a saltación. Sin embargo, según Sheridan se asociarían a balística y a tracción, y a rodamiento/ saltación y saltación, respectivamente. Para el Grupo 2, Wohletz asociaría los procesos 2 y 3 con tracción, y los 4 y 5 con saltación, en tanto que para Sheridan los procesos 1 y 2 serían balísticos, y los 4 y 5 serían ejemplos de saltación.

Según se observa en el cuadro 8,7 de las 10 relaciones tienen asociadas valores de $\mathrm{R}^{2}$ mayores a 0,5 , y para 3 de esas 7 el valor de $R^{2}$ es muy cercano a la unidad, lo que sugiere que reflejan uno o varios fenómenos físicos presentes durante el transporte de los fragmentos, pues se han generado partiendo de muestras localizadas en diversos lugares, recolectadas en tiempos y en condiciones bastante diferentes. En el Cuadro 8 se observa todas las pendientes e interceptos son valores negativos entre 0 y la unidad. Además, los asociados a los procesos 2, 3 y 4 son muy parecido, de manera similar a lo que se observa en el gráfico de coeficiente de fragmentación versus moda (Figura 6b).
EnBrenes(2013)sepropuso, comoalternativa, utilizar el gráfico del coeficiente de fragmentación versus la moda para asociar correctamente cada sub-población a una distribución de Weibull, y por ende a un proceso de transporte determinado, buscando las asociaciones que den como resultado ajustes lineales con el $\mathrm{R}^{2}$ más alto posible. De esta manera, se determinó que si la muestras Tur12-315-1 (moda 2,67 $\Phi$ ) y la Tur12-3-15-3 (moda 2,54 $\Phi)$ se asocian al proceso 5, el ajuste lineal muestra un $\mathrm{R}^{2}$ de 0,3819 . Si se asocia con el proceso 4 , en cambio, el ajuste lineal del proceso 5 aumenta significativamente, pasando a $\mathrm{R}^{2}=0,513$, y el del proceso 4 también aumenta de 0,9262 a 0,9515 .

Llama la atención que el mismo resultado se obtiene en el gráfico de $\Delta$ versus $\gamma$. La recta asociada al Proceso 4 muestra un $\mathrm{R}^{2}$ de 0,1904 si las dos muestras se asocian al proceso $5\left(\mathrm{R}^{2}\right.$ $=0,2176)$. Si en cambio las muestras se asocian al proceso 4 , su $\mathrm{R}^{2}$ aumenta apreciablemente a 0,6752 , en tanto que el $\mathrm{R}^{2}$ del proceso 5 apenas disminuye a 0,1831 .

Aunque estas observaciones sugieren fuertemente que el gráfico de $\Delta$ versus $\gamma$ refleja la misma realidad que el de coeficiente de fragmentación versus moda propuesto por Wohletz (1989), del primero se puede obtener mayor información cuantitativa pues, como se mostrará a continuación, la pendiente de los ajustes lineales es una 
Cuadro 8

Resumen de los valores de pendiente e intercepto para $\Delta=\mathrm{m} \gamma+\mathrm{b}$, así como al tipo de transporte. Rod-Sal indica que tanto el Rodamiento como la Saltación son posibles según Sheridan et al. (1987).

\begin{tabular}{ccccccccc}
\hline Grupo & Proceso & $\begin{array}{c}\text { Pendiente } \\
(\mathrm{m})\end{array}$ & $\begin{array}{c}\text { Intercepto } \\
(\mathrm{b})\end{array}$ & $\mathrm{R}^{2}$ & $\begin{array}{c}\text { Según } \\
\text { Sheridan }\end{array}$ & $\begin{array}{c}\text { Según } \\
\text { Wohletz }\end{array}$ & $\begin{array}{c}\text { Ámbito } \\
\text { en } \Phi\end{array}$ & $\begin{array}{c}\text { Ámbito en } \\
\mathrm{mm}\end{array}$ \\
\hline 2 & 1 & $-0,073$ & $-0,173$ & 0,104 & Balística & Balística & $-4,6$ a $-3,4$ & 24 a 10 \\
1 & 1 & $-1,054$ & $-0,873$ & 0,984 & Balística & Tracción & $-3,5$ a $-2,1$ & 11,3 a 4,3 \\
2 & 2 & $-0,399$ & $-0,389$ & 0,551 & Balística & Tracción & $-2,4$ a 0,8 & 5,3 a 0,6 \\
1 & 2 & $-0,392$ & $-0,378$ & 0,950 & Tracción & Tracción & -1 a 0,9 & 2 a 0,5 \\
2 & 3 & $-0,292$ & $-0,298$ & 0,639 & Tracción & Tracción & $-0,5$ a 1,9 & 1,4 a 0,3 \\
1 & 3 & $-0,304$ & $-0,244$ & 0,204 & Rod-Sal & Saltación & 0,1 a 1,7 & 0,9 a 0,3 \\
2 & 4 & $-0,299$ & $-0,313$ & 0,544 & Rod-Sal & Saltación & 1,2 a 3,1 & 0,4 a 0,1 \\
1 & 4 & $-0,166$ & $-0,216$ & 0,675 & Saltación & Saltación & 1,6 a 1,9 & $0,3-0,3$ \\
2 & 5 & $-1,012$ & $-0,449$ & 0,954 & Saltación & Saltación & 3,3 & 0,1 \\
1 & 5 & $-0,110$ & $-0,194$ & 0,183 & Suspen & Suspensión & 3,3 & 0,1 \\
\hline
\end{tabular}

medida de la interacción entre los fragmentos. Además, para el caso de las muestras Tur12-3-151 y Tur12-3-15-3 se puede deducir que aunque los fragmentos de esas dos muestras fueron transportadas por aire (transporte por suspensión, posiblemente asociado al proceso 5) fueron producidas por otro tipo de proceso.

A pesar de lo diferente de las muestras: el Grupo 1, compuesto por muestras tomadas pocas horas o días luego de la erupción en el 2014; y el Grupo 2, compuesto por muestras recolectadas en el 2015 producto de un gran número de eventos, es posible identificar algunos patrones (Cuadro 9)

Las corrientes de densidad piroclásticas se generan en las columnas de erupciones volcánicas explosivas (Sparks y Wilson, 1976) y en las sedimentaciones de nubes diluidas de ceniza que interactuan con la topografía del terreno (Druitt et al., 2002). Son mezclas de partículas y gas caliente que se pueden propagar por varios kilómetros, y que pueden variar desde una mezcla diluida conocida como oleada hasta un flujo denso granular.

El análisis usando SFT de ambos conjuntos de muestras revela que pueden ser considerados como la suma de 5 procesos diferentes, con modas en ámbitos que se traslapan apreciablemente, lo que sugiere la presencia de una retro-alimentación positiva dependiente del tamaño. Esto sugiere que los procesos de transporte pueden estar determinados por la interacción entre los fragmentos, y la proporción relativa de partículas gruesas y finas, lo que recuerda la clasificación de Geldart (1973). Por ejemplo, es posible distinguir una transición al pasar de partículas de tipo A a partículas tipo B alrededor del tamaño de $180 \mu \mathrm{m}(\Phi=3)$, lo que quizá explique el cambio que se nota en los finos (de $3 \Phi$ a $4 \Phi$ ). La mayoría de las partículas con un tipo B de Geldart tienen tamaños de $150 \mu \mathrm{m}$ $(2,7 \Phi)$ a $500 \mu \mathrm{m}(1 \Phi)$ y densidades de $1.4 \mathrm{a} 4 \mathrm{~g} /$ $\mathrm{cm} 3$ similares a las del magma.

Siguiendo a Wohletz et al. (1998) y Sheridan et al. (1987), el ámbito de $-5 \Phi$ a $-3 \Phi$ estaría asociado a procesos balísiticos. La relación $\Delta_{1 \mathrm{G} 1}=$ $-(1,054 \gamma+0,873)$, con un $\mathrm{R}^{2}=0,984$, sugiere que los fragmentos fueron producidos por unos pocos eventos, quizá jets, con condiciones muy bien definidas y constantes dada su $\mathrm{R}^{2}$ muy cercana a la unidad. La alta pendiente indicaría entonces una fuerte inrteracción entre los fragmentos que caerían muy cerca unos de otros dando lugar a que interactúen con los que previamente ya han caido, haciendo que ambas parte se fragmenten. Así se daría una retroalimentación entre la pendiete y el intercepto. Para los fragmentos del Grupo 2, por el contrario,la expresión $\Delta_{1 \mathrm{G} 2}=-(0,073 \gamma+$ $0,173)$ sugiere que los fragmentos prácticamente no interactuaron entre ellos ni en vuelo ni con 
Cuadro 9

Valores de las diferencias $\Delta=\mathrm{g}-\gamma$ asociadas a cada proceso, para evidenciar posibles patrones.

\begin{tabular}{cccc}
\hline Proceso & $\Delta=\mathrm{m} \gamma+\mathrm{b}$ & $\mathrm{R}^{2}$ & Ambito de $\Phi$ \\
\hline 2 Grupo 1 & $\Delta_{2 \mathrm{G} 1}=-(0,392 \gamma+0,378)$ & 0,950 & -1 a 0,9 \\
2 Grupo 2 & $\Delta_{2 \mathrm{G} 2}=-(0,399 \gamma+0,389)$ & 0,551 & $-2,4$ a 0,8 \\
3 Grupo 1 & $\Delta_{3 \mathrm{G} 1}=-(0,304 \gamma+0,244)$ & 0,204 & $0,1 \mathrm{a} 1,7$ \\
3 Grupo 2 & $\Delta_{3 \mathrm{G} 2}=-(0,292 \gamma+0,981)$ & 0,639 & $-0,5$ a 1,9 \\
4 Grupo 1 & $\Delta_{4 \mathrm{G} 1}=-(0,166 \gamma+0,216)$ & 0,675 & 1,6 a 1,9 \\
4 Grupo 2 & $\Delta_{4 \mathrm{G} 2}=-(0,299 \gamma+0,312)$ & 0,458 & 1,2 a 3,1 \\
5 Grupo 1 & $\Delta_{5 \mathrm{G} 1}=-(0,110 \gamma+0,194)$ & 0,183 & 3,3 \\
5 Grupo 2 & $\Delta_{5 \mathrm{G} 2}=-(1,011 \gamma+0,449)$ & 0,954 & 3,3 \\
\hline
\end{tabular}

los ya en el suelo, debido a que las eyecciones se hicieron en direcciones y condiciones altamente cambiantes $\left(\mathrm{R}^{2}=0,104\right)$.

La igualdad $\Delta_{2 \mathrm{G} 1}=\Delta_{2 \mathrm{G} 2}$, válida para fragmentos en el ámbito $-1 \Phi$ a $1 \Phi$ en dos grupos de muestras generados en ambientes muy diferentes sugiere un mecanismo que funciona con mucho (Grupo 2) o poco material (Grupo 1). La gran similitud entre la pendiente y el intercepto sugiere que el suelo es tan importante como los fragmentos, como podría ser el caso de tracción. La reducción del $\mathrm{R}^{2}$ asociado al Grupo 2 puede deberse a cambios en el tiempo de la composición o la temperatura del magma.

La comparación de $\Delta_{3 \mathrm{G} 1}$ y $\Delta_{3 \mathrm{G} 2}$, válida para fragmentos en el ámbito $0 \Phi$ a $2 \Phi$ en dos grupos de muestras generados en ambientes diferentes, sugiere un mecanismo en que las interacciones entre las partículas son independientes de la cantidad de material (pendientes casi iguales), pero cuya interacción con el sustrato varía con la cantidad de material. El $\mathrm{R}^{2}$ al igual que el intercepto aumentan sensiblemente cuando se incorpora material de varios eventos, lo que sugiere fragmentos en un fluido, de manera que un aumento en su concentración lleva a una uniformización del movimiento. Así, el Grupo 1 se dio en un fluido ralo, en tanto que el Grupo 2 ocurrió en un fluido denso.

La comparación de $\Delta_{4 \mathrm{G} 1}$ con $\Delta_{4 \mathrm{G} 2}$, válida para fragmentos en el ámbito $1 \Phi$ a $3 \Phi$ sugiere un mecanismo en que las interacciones entre las partículas y con el sustrato son dependientes de la concentración de material (pendientes e intercepto varían). Si el transporte en cuestión fuese por saltación, la baja concentración de material en el Grupo 1 causaría que los fragmentos colisionarían en mayor medida con el suelo que con otro fragmento, lo que llevaría a que el intercepto se acerque al valor de 0,17 (Proceso 1) que se obtuvo para las balísticas. Al aumentar la concentración, el intercepto se acercaría al de tracción (Proceso 2). La disminución del $\mathrm{R}^{2}$ observada para el Grupo 2, puede deberse a que la colisión de los fragmentos con los otros que están en el suelo puede ser inelástica, y parte de su energía cinética se disipa como deformación, lo que podría reflejarse en un aumento de la pendiente en $\Delta_{4 \mathrm{G} 2}$. El material del Grupo 1 puede haberse enfriado más rápidamente al estar más expuesto al aire. Las muestras del Grupo 2, si fueron eyectadas más frecuentemente y en direcciones variables puede haber calentado la atmósfera alrededor de los fragmentos, enlenteciendo su enfriamiento.

El Proceso 5 llama la atención por que la expresión de $\Delta_{5 \mathrm{G} 1}=-(0,11 \gamma+0,194)$ con un $\mathrm{R}^{2}=$ 0,183 es bastante similar a la del Proceso 1 pero del grupo $2: \Delta_{1 \mathrm{G} 2}=-(0,073 \gamma+0,173)$ con un $\mathrm{R}^{2}$ $=0,104$, y a la inversa: la expresión de $\Delta_{5 \mathrm{G} 2}=$ $-(1,012 \gamma+0,449)$ con un $\mathrm{R}^{2}=0,952$ es bastante similar a la del Proceso 1 pero del grupo 1: $\Delta_{1 \mathrm{G} 2}$ $=-(1,054 \gamma+0,873)$ con un $\mathrm{R}^{2}=0,984$. De acuerdo con los experimentos reportados en Gladstone et al. (1998) la adición de una pequeña cantidad de partículas finas (similar a lo que se obtendría si una porción de esas partículas se fragmentara secuencialmente) a una corriente de partículas gruesas tiene un mayor efecto en la velocidad del 
flujo y en los patrones de sedimentación que aquel que se produce al añadir una pequeña cantidad de partículas gruesas a una corriente de partículas finas (como podría suceder si por transporte balístico se añade material a una corriente de densidad). Además, la gran mayoría de los casos que presentan una fragmentación secundaria están asociados al Proceso 5, lo que sugiere una conminución y liberación de gas de clastos presurizados (Sparks et al., 1978; Fujii y Nakada, 1999; Dufek y Manga, 2008), lo que también podrían dar como resultado una fluidización.

Roche et al. (2004, 2008) investigaron la propagación y deposición del end-member denso de las corrientes piroclásticas de densidad, en tres ambientes bien definidos: flujo en agua (W), flujo en aire (D), y un flujo de partículas finas inicialmente fluidizadas (F). Los resultados muestran que $\mathrm{W}$ requiere mayor tiempo que $\mathrm{F}$ para alcanzar el reposo. La D aunque claramente se distingue de los otros dos ambientes, muestra una velocidad similar. Bajo condiciones apropiadas (¿casos $\Delta_{1 \mathrm{G} 2}$ y $\Delta_{5 \mathrm{G} 1}$, por la pequeña pendiente?) es posible que el transporte se de como un flujo Newtoniano sin viscosidad (Roche, 2004). La fluidización pudo deberse a la elutriación de finos de la "basal avalanche" (Fisher, 1979; Wilson, 1980) o bien por la difusión turbulenta a traves de una capa límite (Denliger, 1987; Burgisser y Bergantz, 2002). En Druitt et al. (2002) se presenta el caso de una alta movilidad causada por una rápida sedimentación, y en Kokelaar et al. (2004) se reporta una elutriación de finos durante el proceso de transporte.

Estas consideraciones sugieren que los procesos de transporte detectados por SFT están determinados por números adimensionales (del tipo Savage, Sa, y Reynolds, Re) en diversas combinaciones, según uno o varios de ellos sea mayor o menor que el correspondiente valor crítico asociado.

Estas observaciones sugieren que quizá el análisis de los procesos de transporte por tracción, rodamiento y saltación es incompleto pues se basa en experimentos en que el material no se fragmenta mientras es transportado (la llamada fragmentación secuencial), posibilidad que ahora si se contempla. La pendiente en la relación $\Delta=\mathrm{m} \gamma+\mathrm{b}$ incorpora como varía la dispersión por transporte g conforme varía $\gamma$, y sería una medida de la interacción entre las partículas transportadas por la corriente de densidad, lo que recuerda al esfuerzo por dispersión propuesto en Bagnold (1954) o el concepto de contactos por colisiones presentados en Iverson (1997).

En resumen, el análisis de los resultados sugiere que las muestras fueron recolectadas en una parte en que el material se transportaba como una mezcla de una parte oleada diluida sobrepuesta en una corriente granular. Por ende, el depósito está dominado por las colisiones entre las partículas y los esfuerzos cortantes altos (número de Savage mayor a 0,1) (Savage y Hutter, 1989). De ser así, se esperaría que para muestras recolectadas más lejos de la fuente el número de Reynolds asociado se reduciera, pasándose de un flujo turbulento a uno laminar. Las partículas suspendidas en el flujo debido a la turbulencia podrán entonces irse depositando, variando el contraste de densidad de los dos flujos (Valentine, 1987; Dellino et al, 2008)

El número de Reynolds puede ser útil si hay equivalencia hidráulica, que en lo medular significa que las condiciones que permitan la deposición de un grano de cierto tamaño también darán lugar a la deposición a la misma velocidad de un grano más pesado pero de un tamaño menor. Este principio no aplica en casos en que el material granular no es cohesivo por las interacciones y complejidades en el tamaño, densidad, forma y tipo de transporte. La imposibilidad puede deberse tanto a restricciones en el tamaño, propias del material, o bien en el entrainment diferencial de los granos (Lowright et al. ,1972; Grazanti et al., 2008).

\section{CONCLUSIONES}

El método SFT en la parte de fragmentación, representada por la dispersión $\gamma$, ha sido pocas veces utilizado para analizar erupciones volcánicas, a pesar de su base física. La parte de transporte, representada por la dispersión 
por transporte $\mathrm{g}$, también ha sido muy pocas veces utilizada (Wohletz et al., 1987; Brenes y Alvarado, 2013).

En este trabajo buscó analizar más a profundidad la nueva dispersión g propuesta en Wohletz et al. (1989). Para ello, se analizaron los valores de dispersión por transporte g calculada a dos grupos de muestras de las erupciones del volcán Turrialba (2010 a 2014), sensiblemente diferentes.

La asociación de tipos de transporte con ayuda de los parámetros de Folk generó resultados contradictorios. La incorporación del SFT por medio de la substitución de la desviación estandard de Folk por el valor promedio de g generó igualmente resultados inconsistentes, sugiriendo que la lógica del SFT obliga a desagregar cada una de las granulometrías en diversos procesos, cada uno de los cuales deberían ser asociados a uno de los tipos de transporte. Más aún, la definición de la dispersión por transporte $\mathrm{g}$ obliga a asociar la diferencia $\Delta=\mathrm{g}-\gamma$, más que la $\mathrm{g}$ promedio, al tipo de transporte específico.

La observación directa de las corrientes de densidad piroclástica es muy difícil, por lo que sus propiedades deben ser inferidas de los depósitos. Por ello la relación empírica $\Delta$ $=\mathrm{m} \gamma+\mathrm{b}$, y los posibles patrones que puedan observarse se vuelven una herramienta valiosa para estudiarlas. De los patrones entre la mayoría de las $\Delta$ asociadas a cada uno de los 10 procesos identificados, sugieren la pendiente $\mathrm{m}$ es una medida de la intensidad de las colisiones entre las partículas, y el intercepto b sea una medida de la interacción con el sustrato. Un estudio sistemático de estas ideas en otros escenarios, junto con las propiedades de números adimensionales como el de Savage, o el de Reynolds, podrían ayudar a deducir algunas propiedades de los procesos de las corrientes de densidad.

Los experimentos efectuados en los últimos años, que han llevado a aceptar que el colapso de partículas en un medio gaseoso tiene muchas cosas en común con el que se da en un medio líquido (Lube et al., 2004; Lajeunesse et al., 2004; Roche et al., 2008), unido al hecho de que el SFT en 'principio no está restringido al análisis de granulometrías de origen volcánica, implican que el nuevo análisis aquí presentado puede extenderse a lahares, flujos turbídicos en el mar, flujos estuarinos, y otros en que el concepto de corriente de gravedad sea aplicable.

\section{REFERENCIAS BIBLIOGRÁFICAS}

Alvarado, G. E., Brenes-André, J., Barrantes, M., Vega, E., De Moor, M., Avard, G., Dellino,P., Mele, D., De Vitre, C., Di piazza, A., Rizzo, A. L. y Carapezza, M. L. (2016). Esclareciendo la actividad eruptiva del Turrialba (Costa Rica) en el 2010-2015. Revista Geológica de América Central, 55, 7-58

Alvarado, G. E., Soto, G. J., Schminke, H-U., Bolge, L. L. y Sumita, M. (2006). The 1968 andesitic lateral blast eruption at Arenal Volcano, Costa Rica. Journal of Volcanological and Geothermal Research, 157, 9-33.

Arthur, J., Applegate, J., Melkote, S. y Scott, T. (1986). Heavy mineral reconnaissance off the coast of the Apalichocola River delta, Northwest Florida. Florida Geological Survey, Report of Investigation, 95.

Bagnold, R. A. (1954). Experiments on a gravityfree dispersion of large solid spheres in a newtonian fluid under shear. Proceedings of the Royal Society of London, A225, 4963.

Brenes, J. (2013). Aplicación de la teoría de fragmentación/transporte secuencial a los depósitos de las erupciones de 1723 y 1963-65 del volcán Irazú, Costa Rica. Caso dispersión negativa. Revista Geológica de América Central, 48, 63-85.

Brenes, J. y Alvarado, G. E. (2013). Aplicación de la teoría de fragmentación/transporte secuencial a los depósitos de las erupciones de 1723 y 1963-65 del volcán Irazú, Costa Rica. Caso de dispersión positiva y mode- 
lo fractal. Revista Geológica de América Central, 48, 87-98.

Brenes-Andre, J. (2016). Modelo fractal de una erupción aplicado a volcanes denominados log-logísticos: Colima, Soufriere y Erebus.- Revista Geológica de América Central, 55, 59-67.

Brown, W. (1984). A theory of sequential fragmentation and its astronomical applications.- Journal of Astrophysics and Astromy, 10, 89-112.

Burgisser, A. y Bergantz, G. W. (2002). Reconciling pyroclastic flow and surge: the multiphase physics of pyroclastic density currents.- Earth and Planetary Science Letters, 202: 405-418.

Cas, R. A. F. (1989). Physical volcanology in Australian and New Zealand Cainozoic intraplate terrains. En R. W. Johnson (ed.), Intraplate Volcanism in Eastern Australia and New Zealand (pp. 55-85). Cambridge University Press, pp 408.

Cas, R. A. F. y Wright, J. V. (1987). Volcanic successions: Modern and ancient: $A$ geological approach to processes, products and successions. London: Unwin Hyman Inc., Allen and Unwin Ltd.

Delinger, R. P. (1987). A model for generation of ash clouds by pyroclastic flows, with appplications to the 1980 eruptions of Mount St. Helens, Washington. Journal of Geophysical Research, 92, 284-298.

Dellino, P., Mele, D., Sulpizio, R., La volpe, L. y Braia, G. (2008). A method for the calculation of the impact parameters of dilute pyroclastic density currents based on deposit particle charactersitics. Journal of Geophysical Research, 113, B07206.

Dellino, P., Isaia, R., La volpe, L. y Orsi, G. (2004a). Interaction between particles transported by fallout and surge in the deposits of the Agnano-Monte Spina eruption (Campi Flegrei, Souther Italy). Journal of Volcanology and Geothermal Research, 133, 193-210.

Dellino, P., Isaia, R. y Veneruso, M. (2004b). Turbulent boundary layer shear flows as an approximation of base surges at Campi Flegrei (Southern Italy). Journal of Volcanology and Geothermal Research, 133, 211-228.

Doeglas, D. J. (1946). Interpretation of the results of mechanical analysis. Journal of Sedimentary Petrology, 16, 19-40.

Druitt, T. H., Calder, E. S., Cole, P. D., Hoblitt, R. P., Loughin, S. C., Norton, G. E., Ritchie, L. J., Sparks, R. S. J. y Voight, B. (2002). Small volume, highly mobile pyroclastic flows formed by rapid sedimentation from pyroclastic surges at Soufrière Hills Volcano, Montserrat: An important volcanic hazard. Memoirs of the Geological Society of London, The Eruption of Soufrière Hills Volcano, Montserrat, from 1995 to 1999, 21, 263 279.

Dufek, J. y Manga, M. (2008). In situ production of ash in pyroclastic flows. Journal of Geophysical Research, 113, 1-17.

Eschner, T. R. y Kircher, J. E. (1984). Interpolation of grain-size distributions from measured sediment data, Platte River. Sedimentology, 31, 569-573. 
Fisher, R. V. (1979). Models for pyroclastic surges and pyroclastic flows. Journal of Volcanology and Geothermal Research, 6, 305-318.

Fujii, T. y Nakada, S. (1999). The 15 September 1991 pyroclastic flows at Unzen Volcano (Japan): a flow model for associated ashcloud surges. Journal of Volcanology and Geothermal Research, 89, 159-172.

Geldart, D. (1973). Types of gas fluidization, Powder Technology, 7, 285-292.

Grazanti, E., Ando, S. y Vezzoli, G. (2008). Settling equivalence of detrital minerals and grain-size dependence of sediment composition. Earth and Planetary Science Letters, 273, 138-151.

Iverson, R. M. (1997). The physics of debris flows. Reviews of Geophysics, 35, 245-296.

Kokelaar, B. P., Grahama, R. L., Gray, J. M. N. T. y Vallance, J. W. (2004). Fine-grained linings of leveed channels facilitate runout of granular flows. Earth and Planetary Science Letters, 385, 172-180.

Lajeunesse, E., Mangeney-Castelnau,a. y Vilotte, J.P. (2004). Spreading of a granular mass on a horizontal plane. Physics of fluids, 16, 2371-2381.

Lowright, R., Wlliams, E.G. y Dachille, F. (1972). An analysis of factors controlling deviations in hydraulic equivalence in some modern sands. Journal of Sedimentary Petrology, 42, 635-645.

Lube, G., Huppert, H. E., Sparks, R. S. J. y Hallworth, M. A. (2004). Axisymmetric collapses of granular columns. Journal of Fluid Mechanics, 508, 175-199.
Roche, O., Gilbertson, M. A.; Phillips, J.C. y Sparks, R. S. J. (2004). Experimental study of gas-fluidized granular flows with implications for pyroplastic flow emplacemen. Journal of Geophysical Research, 109, B10201.

Savage, s.B. Y hutter, K., 1989: The motion of a finite mass of granular material down a rough incline. Journal of Fluids Mechanics, 199, 177-215.

Sheridan, M. F., Wohletz, K. H. y Dehn, J. (1987). Discrimination of grain-size subpopulations in pyroclastic deposits. Geology, 15, 367-370.

Sheridan, M. F. (1971). Particle-size characteristics of pyoroclastic tuffs. Journal of Geophysical Research, 76, 5627-5634.

Soto, J. y Alvarado, G. E. (2006). The eruptive history of Arenal volcano, Costa Rica, 7ka to present. Journal of Volcanology and Geothermal Research, 157, 254-269.

Sparks, R. S. J. y Wilson, L. (1976). A model for the formation of ignimbrite by gravitational column collapse. Journal of the Geological Society of London, 132, 441-451.

Sparks, R. S. J., Wilson, L. y Hulme, G. (1978). Theoretical modeling of generation, movement, and emplacement of pyroclastic density flows by column collapse. Journal of Geophysical Research, 83, 1727-1739.

Spera, J. (1984). Some numerical experiments on the withdraw of magma from crustal reservoirs. Journal of Geophysical Research, 89, 8222-8236. 
Swan, D., Clague, J. J. y luternauer, J. L. (2006). Grain-size statistics I; Evaluation of the Folk and Ward graphic measures. Journal of Sedimentary Research, 48, 863-878.

Valentine, G. A. (1987). Stratified flow in pyroclastic surges. Bulletin of Volcanology, 49, 616-630.

Visher, G. S. (1969). Grain-size distributions and depositional processes. Journal of Sedimentary Petrology, 39: 1074-1106.

Wilson, C.J.N., 1980:- The role of fluidization in the emplcement of pyroclastic claws: an epe- rimental approach. Journal of Volcanology and Geothermal Research, 8, 231-249.

Wohletz, K. (1998). Pyroclastic surges and compressible two-phase flow. En A. Freundt y M. Rosi (eds), From magma to tephra: modelling physical processes of explosive volcanic eruptions (pp. 247-312). Amsterdan: Elsevier.

Wohletz, K. H., Sheridan, M. F. y Brown, K. (1989). Particle size distribution and the Sequential Fragmentation/Transport Theory Applied to Volcanic Ash. Journal of Geophysical Research, 94, 15 703-15 721 . 